\title{
Evaluation of the v2.0 Brief Profiles for Sexual Function and Satisfaction PROMISR in adolescent and young adult childhood cancer survivors
}

\author{
Jenna Sopfe ${ }^{1}$, Rebekah Marsh ${ }^{1}$, Sonja Ziniel ${ }^{1}$, James Klosky², Eric Chow ${ }^{3}$, Brooke Dorsey \\ Holliman $^{1}$, and Pamela Peterson ${ }^{1}$ \\ ${ }^{1}$ University of Colorado Denver School of Medicine \\ ${ }^{2}$ Emory Healthcare \\ ${ }^{3}$ Fred Hutchinson Cancer Research Center
}

September 11, 2020

\begin{abstract}
Background: Sexual dysfunction (SD) is a common but often unrecognized potential late effect among childhood cancer survivors (CCS). Unfortunately, both patients and providers report low levels of routine screening and identify multiple barriers, including lack of knowledge, discomfort, and limited time, particularly among CCS who are adolescent or young adult aged (AYA-CCS). One potential way to increase screening, detection and treatment for SD among AYA-CCS is to employ patient-reported outcomes measures. While adult screening tools exist, no SD screening tool has been evaluated specifically among this younger population. Procedure: This qualitative study used Think-Aloud and cognitive interviewing methods to obtain feedback from

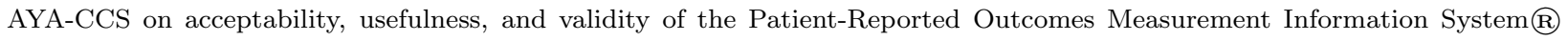
(PROMIS®) v2.0 Brief Profiles for Sexual Function and Satisfaction (SexFS Brief) in CCS now aged 15-24 years. Results: The SexFS Brief demonstrated acceptability, response process and content validity, and usefulness among AYA-CCS. There were no detectable differences by age or gender. This study did not reveal any necessary modifications to the SexFS Brief for this population. Conclusions: The PROMIS SexFS Brief is an acceptable and useful tool, with demonstrated response-process and content validity, and may facilitate improved screening and diagnosis of SD among AYA-CCS. Further, this tool was viewed favorably by AYA-CCS as a way to reduce barriers such as discomfort and lack of knowledge on the part of patients. Further evaluation of its effectiveness and acceptability in a clinical setting are warranted.
\end{abstract}

\section{Introduction}

Sexual dysfunction (SD) is estimated to occur in 20-50\% of childhood cancer survivors (CCS), but is widely underrecognized because SD screening rarely occurs. ${ }^{1-10} \mathrm{SD}$, which encompasses features such as lack of desire, failed arousal, or pain with sex, can occur in this population as a result of physiologic or psychosexual reasons, such as poor body image, concerns about fertility, and disruption of normal development. ${ }^{1,2,10-12}$ Both patients and providers report low levels of routine screening and acknowledge multiple barriers, including lack of knowledge, discomfort, and limited time. ${ }^{13-17}$ In one study, all adolescent and young adult-aged CCS reported inadequate clinical support and in another, only $2.9 \%$ of adult CCS experiencing SD had ever received an SD-directed intervention; as such, efforts to improve detection and treatment of SD are necessary. ${ }^{10,13,14}$

Given the sensitivity of this topic, screening tools, or patient-reported outcome (PRO) measures, may be particularly useful in CCS who are now adolescent or young adult-aged (hereinafter, AYA-CCS), who may be especially vulnerable to discomfort related to discussions of sexuality and sexualfunction. ${ }^{16,18-20}$ However, 
no SD-specific PROs have been specifically validated in the AYA general or AYA-CCS population. ${ }^{20}$ The Patient-Reported Outcomes Measurement Information System ${ }^{\circledR}\left(\right.$ PROMIS $\left.{ }^{\circledR}\right)$ v2.0 Brief Profiles for Sexual Function and Satisfaction (SexFS Brief) is a short, gender-specific PRO which has been validated in adult (mean age 58.5 years) cancer populations. ${ }^{9,21,22}$ While these studies do demonstrate validity down to age 18 , only $7 \%$ were $<40$ years old.

Emerging adulthood (age 15-25) or youthhood (age 15-24), is a period of transition, during which individuals may be more vulnerable or unique in their SD concerns. ${ }^{16,23,24}$ Arnett identified five features of this developmental stage: identify exploration, instability, self-focus, feeling in-between and possibilities/optimism. ${ }^{25}$ Because this is such a unique life stage, evaluating use of the SexFS Brief specifically in this population is warranted.

This qualitative study sought to evaluate novel use of the PROMIS SexFS Brief in adolescent CCS $(<18$ years), as well as to confirm acceptability, response process and content validity and usefulness specifically in emerging adult CCS (18-24 years).

\section{Methods}

\section{Study Design}

This qualitative study used Think-Aloud and cognitive interviewing methods to obtain feedback from AYACCS on acceptability, usefulness, and validity of the PROMIS SexFS Brief for this population. ${ }^{26-30}$ These methods are evidence-based qualitative approaches for assessing respondent understanding and content validity, and have been used both in creation of surveys and in evaluating existing surveys for new popultions. ${ }^{26-30}$ Specifically, we used the Think-Aloud approach, in which participants completed the Sex FS Brief while actively verbalizing their thoughts. ${ }^{31}$ Use of Think-Aloud allows a glimpse of participants' thought processes during use of the SexFS Brief, including immediate impressions and problems or questions encountered in understanding or answering the survey item. After undergoing Think-Aloud data collection, participants underwent cognitive interviewing to evaluate whether the survey fulfilled its intended purpose, with specific probes to elucidate acceptability, content validity, and usefulness. ${ }^{32}$ This study was performed after obtaining local institutional review board approval. In addition to qualitative data collection, basic demographic, diagnostic and treatment information were also collected.

\section{Participants and Setting}

Participants were recruited from survivorship clinics at an academic children's hospital and an academic cancer center. Inclusion criteria for participation were as follows: 1) History of cancer diagnosed prior to age o18; 2) Aged 15-24 years at time of study; 2) Treatment consisting of chemotherapy and/or radiation therapy; 3) Completion of therapy a minimum of two years prior to study participation; and 4) English-speaking and reading (due to PROMIS SexFS Brief availability). Patients who did not have sufficient cognitive functioning to participate in all study measures, as determined by their clinician, were excluded. The age range of 15-24 was selected based on the World Health Organization (WHO) and United Nations Educational, Scientific and Cultural Organization (UNESCO) definitions for "youth". ${ }^{23,24}$ This age range also near-mirrors previous studies evaluating barriers to communication in this specific population. ${ }^{16}$

Due to confidentiality concerns, a waiver of parental consent was obtained; thus, eligible patients were enrolled and self-consented, regardless of age. Recruitment was completed consecutively through existing clinic visits; to achieve maximum thematic variation across age and gender (self-identified), we purposefully enrolled a minimum of 10 patients of each gender (self-identified) and a minimum of 10 patients in each of two age groups (15-19 and 20-24 years). Upon the completion of the interview, each participant received a $\$ 50$ gift card.

\section{Measure}

The National Institute of Health-developed PROMIS tools are a set of person-centered measures to evaluate various physical, mental and social health concerns across many populations. The PROMIS Sexual Function 
and Satisfaction (SexFS) tools are a set of measures aimed at assessing sexual function and satisfaction. These tools have gender-specific coordinating versions and have been validated in adult (mean age 58.5 years) cancer populations. ${ }^{21,22}$ The PROMIS SexFS includes multiple validated (content validity, face validity, construct validity and reliability) modules that can be selected to obtain detailed information about various types of SD. Specifically, in adult cancer populations, the PROMIS SexFS tools demonstrate high internal consistency (Cronbach's alpha 0.87-0.95) and favorable test-retest reliability $(0.71-0.87) .{ }^{21}$ In this study, we evaluated the PROMIS v.2.0 Brief Profile SexFS, which was created to be a clinically useful general screening tool and consists of 10 questions for males and 14 questions for females. ${ }^{9,21,22}$ As described below, an additional set of similarly formatted questions on a different topic, anxiety, were used for a practice round.

PROMIS measures were administered and managed using REDCap electronic data capture tools hosted at the University of Colorado Denver. ${ }^{33}$ REDCap (Research Electronic Data Capture) is a secure, web-based application designed to support data capture for research studies. In contrast to the intended presentation of the SexFS Brief each item was presented individually to facilitate Think-Aloud.

\section{Procedures}

One-on-one interviews were conducted via phone by trained research assistants who were matched in gender to the patient. Interviews were digitally recorded and professionally transcribed; additionally, detailed field notes were taken during and immediately after the interviews. Each interview was roughly 30-60 minutes long and had three parts. The first part consisted of qualitative interviews about patient preferences for discussing SD, and these results will be published separately. The second part consisted of Think-Aloud methods and the third part consisted of cognitive interviewing. Per best practice recommendations, prior to completing Think-Aloud procedures using the SexFS Brief, participants received an introduction to the exercise and participated in a practice Think-Aloud exercise with questions derived from an unrelated PROMIS measure with similar formatting. ${ }^{31}$ Specifically, patients were asked to state the question number and to verbalize all thoughts as they answered each question. This practice procedure served to familiarize participants with the Think-Aloud method and to allow them to clarify any misunderstandings about the task. In both the practice and SexFS Brief rounds, participants were not interrupted; occasional reminders to verbalize their thoughts were used when needed, as well concurrent and spontaneous verbal probing to explore any verbal or nonverbal behavior such as hesitations or uncertainty (i.e. pauses, or vocalizations such as sighs or "Ummm"). At the conclusion of the SexFS Brief, patients were advised not to submit their responses to the questions in order to provide further confidentiality, as collecting survey responses was not the purpose of this study.

Following the Think-Aloud task, additional cognitive interviewing was performed. Probes were both semistructured and reactive. In accordance with evidence-based cognitive interviewing for survey evaluation, probes addressed each PRO item and included general questions such as "Was that easy or hard to answer?", or "I noticed that you hesitated. Tell me about that", ${ }^{32,34}$ Focused probes evaluated response process validity according to Tourangeau's 4-stage cognitive model for answering questions (understanding, retrieval, judgement, and response). ${ }^{35}$ In addition, comprehensive participant impressions of the content validity of the SexFS Brief were evaluated with questions such as "What do you think about this questionnaire?", "Were there any questions that didn't feel relevant to you?", and "Was there anything not included that you thought it should have asked?". ${ }^{34}$ Finally, probes evaluated participant overall impressions of acceptability and usefulness with questions such as "How was completing that survey for you?".

\section{Analysis}

Content analysis methodology guided data interpretation. ${ }^{36}$ This approach uses characteristics and content of language to provide understanding of qualitative data through systematic classification, coding, and theme identification. ${ }^{36} \mathrm{~A}$ team-based, inductive process was conducted, beginning with data immersion. Two researchers independently reviewed an initial set of transcripts to inductively identify a set of codes. Codes were organized both by overall impressions of the tool and by specific SexFS Brief item. Subsequently, the research team met to review respective code lists and transcripts, reconciling any discrepancies in the code definitions and applications. This process continued until a final codebook was established and the research 
team had double-coded $20 \%$ of transcripts. The remainder of transcripts was coded by a single member of the research team. Coded transcripts were entered into ATLAS.ti (Version 8) for data management.

Codes were then clustered into related categories for theme development, informed by prior Think-Aloud studies. ${ }^{37-39}$ Coded data was analyzed within codes, by PROMIS item number, and by gender and age group (15-19 and 20-24) to identify themes and variation across subgroups. This was followed by creation of an overall framework of tool acceptability, usefulness, and validity, again with consideration of differences by age and gender.

\section{Results}

Of a total of 46 eligible participants approached, 24 participated in interviews (52\%). The median age was 21 years (range 15-24) with $10(41.7 \%)$ patients in the younger (15-19 years) group and 14 patients in the older (20-24 years) group. Ten (41.7\%) were male and 14 were female and all patients reported congruence between chosen gender and assigned sex. The majority of patients were White and non-Hispanic. Additional demographic, diagnostic and treatment data are reported in Table 1.

Below, we report the results of SexFS Brief acceptability, usefulness and validity, using de-identified quotes from various participants that further describe and contextualize these properties.

\section{Overarching acceptability and usefulness}

Patients widely reported that completing the SexFS Brief was "comfortable" and "straightforward". Representative quotations are shown in Table 2. Rare reports of discomfort with the PRO tool were reported, but generally with the accompanying acknowledgement that the questions were still important.

Multiple patients, particularly in the age 20-24 age group, expressed a desire to have the SexFS Brief used in clinical settings, with one stating, "These are really important questions to be asked and to give real answers... I think that if this tool could be applied, could be really cool" (Female, age 24) (Table 2). Only one participant noted that she would prefer in-person conversations over the use of the SexFS Brief. Others expressed that the SexFS Brief would be useful in filling a needed gap in care; for example, a different participant stated, "They were all questions that I wish someone would've asked me" (Female, age 22). Some participants, across both age groups, stated that completing the SexFS Brief helped them to think of questions they should be asking their physician about, but hadn't previously thought to ask; for example, one stated that it "made [him] curious if any erectile dysfunction was a result of the treatment [he] had" (Male, age 24).

\section{Response process validity}

Basic response process validity, including participant ability to comprehend, recall, make a judgement about the recalled information, and find and select a response to the question, was qualitatively demonstrated throughout the study. Specifically, participants universally reported that items were clear and easy to understand and were observed to appropriately provide answers based on Think-Aloud data, demonstrating comprehension (Table 3). Occasionally, participants reported that they were unclear about terms such as "pain" versus "discomfort" and "vagina" versus "vulva" initially, but understood the term once seeing its counterpoint in a subsequent question. Because typically the SexFS Brief would present all questions at once as a scale, participants would be able to return to a prior question; thus, changes to the instrument were not felt to be necessary.

Participants did not verbally or nonverbally (i.e. through vocalizations such as "Hmmm" or pauses) demonstrate difficulty with recall of their own experiences. They also reported comfort with answering questions truthfully (judgement/estimation), with several even stating that they "expected a lot worse". Similarly, there was minimal difficulty observed or reported in participants' ability to select an appropriate response. However, there were a few participants who mentioned a preference for more concrete, quantitative answer choices instead of choices like "Occasionally" and "Some of the time".

Content validity 
The SexFS Brief demonstrated adequate patient-reported content validity, meaning participants felt that the questions were relevant to SD and all SD domains were included (representative quotations presented in Table 3). Specifically, nearly all participants reported that the questions uniformly felt relevant and important to SD. For example, one participant recognized relevance both to others and to himself: "... the ones about how often and stuff definitely [are] relevant for everybody... yeah, I guess I connected with it" (Male, age 17). Another participant acknowledged the importance of questions to SD, stating "these are really important questions to be asked and to give real answers to" (Female, age 24). When explicitly asked about questions that are missing from the SexFS Brief, several potential questions were mentioned, but none by more than one participant. These singularly mentioned potential missing items were questions on gender identity, sex drive, vaginal lubrication, breast pain, concerns or anxiety about SD due to cancer history, and effects of cancer therapy on puberty.

\section{Subgroup analyses}

As previously mentioned, members of the 20-24 age group were more expressive of the usefulness of the SexFS Brief; this sentiment was not contradicted by the 15-29 age group. No other notable differences by age group were noted.

Across genders, while females tended to be more descriptive of their impressions of the SexFS Brief, there were no obvious differences in the content of impressions.

\section{Discussion}

This cognitive interview study demonstrates that the PROMIS SexFS Brief is an acceptable and useful too, demonstrating response process and content validity, for screening for sexual dysfunction in adolescent and young adult childhood cancer survivors as young as age 15. Most participants in this study were in favor of using this tool to both screen and facilitate conversations about SD in pediatric oncology/survivorship settings. Participants largely reported that the tool was comprehensive and relevant, and appreciated its detailed questions even when a question generated discomfort. While a number of additional questions were suggested by participants, there were no questions felt to be missing by more than one participant. Questions were clear and understandable, although occasional participants advocated for more concrete response options. Importantly, these findings did not appear to differ across age group or gender. As previously mentioned, this is the first study to specifically evaluate an SD screening tool in a youth population, both among general youth and CCS. ${ }^{20,40}$ However, these findings are consistent with studies in adult cancer survivor populations that demonstrate reliability and validity of the PROMIS SexFS Brief. ${ }^{21,22}$

In addition to demonstrating response process and content validity, use of the SexFS Brief in a clinic setting was found not only be acceptable, but useful and favored by participants. Participants indicated that the PROMIS SexFS Brief would address an unmet need with regard to SD and sexual health and would help them to think about their own concerns more thoroughly. Additional research to understand AYA-CCS preferences for SD screening, including evaluating AYA-CCS opinions on the benefits of the use of a PRO tool in comparison to the current standard-of-care, in-person conversations, are forthcoming. We are not aware of other studies demonstrating patient-reported acceptability and usefulness of an SD screening tool in this population. However, findings of this study do support the Clinician Toolbox for assessing sexual impacts of cancer among AYA that was published by Aubin, which suggest that use of an SD PRO measure would facilitate conversations and reduce barriers to SD screening. ${ }^{20}$

Numerous studies demonstrate that AYA-CCS report inadequate clinical support for SD, highlighting the pressing need for improved detection and treatment. ${ }^{13,14}$ Use of the SexFS Brief may help to improve detection of SD by overcoming patient- and provider-identified barriers such as lack of comfort, knowledge and time. ${ }^{13-17}$ Additional studies are necessary to more widely establish acceptability, usefulness, feasibility and effectiveness of this screening tool at improving clinical care in the AYA-CCS population, as well as in patients actively undergoing therapy.

This study has several limitations. While our sample size was consistent with recommended guidelines and 
we purposefully sampled across age and gender, not all subgroups could be analyzed. For example, additional evaluation of acceptability and validity of this tool in patients at higher risk for SD, such as patients with treatment-related gonadal dysfunction, or patients treated with pelvic or central nervous system radiation, may be warranted. Notably, however, participants were diverse in terms of cancer diagnosis and treatment exposures, with one third of patients being survivors of CNS tumors, who may have higher SD risk. ${ }^{11}$ Further, nearly all patients were White, non-Hispanic, and gender-conforming; our study was limited further in that we did not collect data on sexual orientation. Along with this homogeneity, we were unable to include CCS who were not cognitively able to complete the screening tool or were not proficient in reading English. Future work to address screening SD in these populations, as well as to evaluate the SexFS Brief in more diverse subpopulations is necessary. Further, while we did not collect reasons eligible patients declined participation in the study, it is possible that there was some self-selection bias such that patients more comfortable with SD may have been more likely to participate; participant compensation was used to help reduce this bias.

In summary, the PROMIS SexFS Brief was viewed favorably by AYA-CCS for its potential in facilitating discussions and screening of SD between survivors and medical professionals. Because the SexFS Brief has been formally validated in adult cancer populations and demonstrated acceptability as well as response process and content validity in our subset of AYA-CCS, efforts should now focus on use in a clinical setting. If effective at increasing detection, treatment and addressing concerns of SD, use of the PROMIS SexFS Brief may ultimately result in improved quality of care for this often-neglected quality of life concern.

\section{Conflicts of Interest}

All other authors declare no competing financial interests.

Acknowledgements

This work was supported by the Pediatric Cancer Research Foundation Emerging Investigator Fellowship fund and the University of Colorado Cancer Center.

Data Sharing

The data that support the findings of this study are available from the corresponding author upon reasonable request.

Correspondence: Jenna Sopfe, Center for Cancer and Blood Disorders, Department of Pediatrics, University of Colorado School of Medicine, 13123 E. $16^{\text {th }}$ Ave, B115, Aurora, Colorado, 80045; email: jenna.sopfe@cuanschutz.edu

\section{References}

1. van Dijk EM, van Dulmen-den Broeder E, Kaspers GJ, van Dam EW, Braam KI, Huisman J. Psychosexual functioning of childhood cancer survivors.Psychooncology. 2008;17(5):506-511.

2. Wettergren L, Kent EE, Mitchell SA, et al. Cancer negatively impacts on sexual function in adolescents and young adults: The AYA HOPE study.Psychooncology. 2017;26(10):1632-1639.

3. Zebrack BJ, Foley S, Wittmann D, Leonard M. Sexual functioning in young adult survivors of childhood cancer. Psychooncology.2010;19(8):814-822.

4. Bober SL, Zhou ES, Chen B, Manley PE, Kenney LB, Recklitis CJ. Sexual function in childhood cancer survivors: a report from Project REACH.J Sex Med. 2013;10(8):2084-2093.

5. Haavisto A, Henriksson M, Heikkinen R, Puukko-Viertomies LR, Jahnukainen K. Sexual function in male long-term survivors of childhood acute lymphoblastic leukemia. Cancer. 2016;122(14):2268-2276.

6. Ritenour CW, Seidel KD, Leisenring W, et al. Erectile Dysfunction in Male Survivors of Childhood Cancer-A Report From the Childhood Cancer Survivor Study. J Sex Med. 2016;13(6):945-954. 
7. Ford JS, Kawashima T, Whitton J, et al. Psychosexual functioning among adult female survivors of childhood cancer: a report from the childhood cancer survivor study. J Clin Oncol.2014;32(28):3126-3136.

8. van Iersel L, Li Z, Chemaitilly W, et al. Erectile Dysfunction in Male Survivors of Childhood Cancer. JAMA Oncol.2018;4(11):1613-1616.

9. Weinfurt KP, Lin L, Bruner DW, et al. Development and Initial Validation of the PROMIS((R)) Sexual Function and Satisfaction Measures Version 2.0. J Sex Med. 2015;12(9):1961-1974.

10. Bjornard KL, Howell CR, Klosky JL, et al. Psychosexual Functioning of Female Childhood Cancer Survivors: A Report From the St. Jude Lifetime Cohort Study. J Sex Med. 2020.

11. Sundberg KK, Lampic C, Arvidson J, Helstrom L, Wettergren L. Sexual function and experience among long-term survivors of childhood cancer.Eur J Cancer. 2011;47(3):397-403.

12. Laumann EO, Paik A, Rosen RC. Sexual dysfunction in the United States: prevalence and predictors. JAMA. 1999;281(6):537-544.

13. Schover LR. Sexual quality of life in men and women after cancer.Climacteric. 2018:1-5.

14. Frederick NN, Recklitis CJ, Blackmon JE, Bober S. Sexual Dysfunction in Young Adult Survivors of Childhood Cancer. Pediatr Blood Cancer. 2016;63(9):1622-1628.

15. Park ER, Bober SL, Campbell EG, Recklitis CJ, Kutner JS, Diller L. General internist communication about sexual function with cancer survivors. J Gen Intern Med. 2009;24 Suppl 2:S407-411.

16. Frederick NN, Revette A, Michaud A, Bober SL. A qualitative study of sexual and reproductive health communication with adolescent and young adult oncology patients. Pediatr Blood Cancer. 2019:e27673.

17. Frederick NN, Campbell K, Kenney LB, Moss K, Speckhart A, Bober SL. Barriers and facilitators to sexual and reproductive health communication between pediatric oncology clinicians and adolescent and young adult patients: The clinician perspective. Pediatr Blood Cancer. 2018;65(8):e27087.

18. Keegan TH, Lichtensztajn DY, Kato I, et al. Unmet adolescent and young adult cancer survivors information and service needs: a population-based cancer registry study. J Cancer Surviv.2012;6(3):239-250.

19. Stinson JN, Jibb LA, Greenberg M, et al. A Qualitative Study of the Impact of Cancer on Romantic Relationships, Sexual Relationships, and Fertility: Perspectives of Canadian Adolescents and Parents During and After Treatment. J Adolesc Young Adult Oncol. 2015;4(2):84-90.

20. Aubin S, Perez S. The Clinician's Toolbox: Assessing the Sexual Impacts of Cancer on Adolescents and Young Adults with Cancer (AYAC).Sex Med. 2015;3(3):198-212.

21. Flynn KE, Lin L, Cyranowski JM, et al. Development of the NIH PROMIS (R) Sexual Function and Satisfaction measures in patients with cancer.J Sex Med. 2013;10 Suppl 1:43-52.

22. Flynn KE, Reeve BB, Lin L, Cyranowski JM, Bruner DW, Weinfurt KP. Construct validity of the PROMIS(R) sexual function and satisfaction measures in patients with cancer. Health Qual Life Outcomes.2013;11:40.

23. World Health Organization (WHO). Orientation Programme on Adolescent Health for Health-care Providers. https://www.who.int/maternal_child_adolescent/documents/pdfs/9241591269_op_handout.pdf. Published 2006. Accessed.

24. United Nations Educational Scientific and Cultural Organization (UNESCO). What do we mean by "youth"? http://www.unesco.org/new/en/social-and-human-sciences/themes/youth/youth-definition/. Published 2018. Accessed.

25. Arnett JJ. Emerging adulthood: the winding road from the late teens through the twenties. New York; Oxford: Oxford University Press; 2004. 
26. Willis G. Cognitive Interviewing: A Tool For Improving Questionnaire Design. 2005.

27. Patrick DL, Burke LB, Gwaltney CJ, et al. Content validity-establishing and reporting the evidence in newly developed patient-reported outcomes (PRO) instruments for medical product evaluation: ISPOR PRO Good Research Practices Task Force report: part 2-assessing respondent understanding. Value Health.2011;14(8):978-988.

28. Kamp K, Wyatt G, Dudley-Brown S, Brittain K, Given B. Using cognitive interviewing to improve questionnaires: An exemplar study focusing on individual and condition-specific factors. Appl Nurs Res. 2018;43:121-125.

29. Reeve BB, McFatrich M, Pinheiro LC, et al. Cognitive Interview-Based Validation of the Patient-Reported Outcomes Version of the Common Terminology Criteria for Adverse Events in Adolescents with Cancer. $J$ Pain Symptom Manage. 2017;53(4):759-766.

30. Reeve BB, McFatrich M, Pinheiro LC, et al. Eliciting the child's voice in adverse event reporting in oncology trials: Cognitive interview findings from the Pediatric Patient-Reported Outcomes version of the Common Terminology Criteria for Adverse Events initiative. Pediatr Blood Cancer. 2017;64(3).

31. Someren MWv, Barnard YF, Sandberg J. The think aloud method : a practical guide to modelling cognitive processes. London; San Diego: Academic Press; 1994.

32. Willis GB, Artino AR, Jr. What Do Our Respondents Think We're Asking? Using Cognitive Interviewing to Improve Medical Education Surveys. J Grad Med Educ. 2013;5(3):353-356.

33. Harris PA, Taylor R, Thielke R, Payne J, Gonzalez N, Conde JG. Research electronic data capture (REDCap)-a metadata-driven methodology and workflow process for providing translational research informatics support. J Biomed Inform. 2009;42(2):377-381.

34. Peterson CH, Peterson NA, Powell KG. Cognitive Interviewing for Item Development: Validity Evidence Based on Content and Response Processes.Measurement and Evaluation in Counseling and Development.2017;50(4):217-223.

35. Tourangeau R, Rips LJ, Rasinski KA. The psychology of survey response. Cambridge, U.K. ; New York: Cambridge University Press; 2000.

36. Hsieh HF, Shannon SE. Three approaches to qualitative content analysis. Qual Health Res. 2005;15(9):1277-1288.

37. Aujla N, Vedhara K, Walker M, Sprigg N. Evaluating a stroke-specific version of the Illness Perception Questionnaire-Revised, using the Think-Aloud method. J Health Psychol. 2018:1359105318781942.

38. McCorry NK, Scullion L, McMurray CM, Houghton R, Dempster M. Content validity of the illness perceptions questionnaire-revised among people with type 2 diabetes: a think-aloud study. Psychol Health.2013;28(6):675-685.

39. van Oort L, Schroder C, French DP. What do people think about when they answer the Brief Illness Perception Questionnaire? A 'think-aloud' study. Br J Health Psychol. 2011;16(Pt 2):231-245.

40. Sopfe J, Gupta A, Appiah LC, Chow EJ, Peterson PN. Sexual Dysfunction in Adolescent and Young Adult Survivors of Childhood Cancer: Presentation, Risk Factors, and Evaluation of an Underdiagnosed Late Effect: A Narrative Review. J Adolesc Young Adult Oncol. 2020.

\section{Hosted file}

TABLE 1 Participant Disease and Treatment Characteristics 8.21.20.docx available at https://authorea.com/users/321737/articles/478841-evaluation-of-the-v2-0-brief-profilesfor-sexual-function-and-satisfaction-promis-in-adolescent-and-young-adult-childhoodcancer-survivors 


\section{Hosted file}

TABLE 2 Acceptability and usefulness of the SexFS Brief 8.21.20.docx available at https: //authorea.com/users/321737/articles/478841-evaluation-of-the-v2-0-brief-profiles-forsexual-function-and-satisfaction-promis-in-adolescent-and-young-adult-childhood-cancersurvivors

\section{Hosted file}

TABLE 3 Patient-reported validity of the SexFS 8.21.20.docx available at https://authorea. com/users/321737/articles/478841-evaluation-of-the-v2-0-brief-profiles-for-sexualfunction-and-satisfaction-promis-in-adolescent-and-young-adult-childhood-cancersurvivors 